\title{
Obtaining an accurate maternal blood pressure: A quality improvement initiative to improve nursing knowledge and confidence
}

\author{
Matthew K. Wagar*1, Jacquelyn H. Adams², Amy Godecker ${ }^{1}$, Kathleen Frigge ${ }^{3}$, Michele Schroeder ${ }^{3}$, Laura Haak ${ }^{3}$, \\ Amy Oetzel ${ }^{3}$, Miranda Pickett ${ }^{3}$, Kathleen M. Antony ${ }^{3}$ \\ ${ }^{1}$ Department of Obstetrics and Gynecology, University of Wisconsin School of Medicine and Public Health, Madison, WI, USA \\ ${ }^{2}$ Division of Maternal Fetal Medicine, University of Wisconsin School of Medicine and Public Health, Madison, WI, USA \\ ${ }^{3}$ Unity Point Health-Meriter, Birthing Center, Madison, WI, USA
}

Received: October 7, 2021

DOI: $10.5430 /$ jnep.v12n4p30
Accepted: November 16, 2021

Online Published: November 28, 2021

URL: https://doi.org/10.5430/jnep.v12n4p30

\begin{abstract}
Background: Inaccurate assessment of maternal blood pressure (BP) contributes to misdiagnosis of hypertension, unnecessary or missed interventions, and maternal morbidity. This study examines obstetric nursing knowledge and confidence in proper assessment of maternal BP before and after an institutional quality improvement project.

Methods: We implemented an online educational initiative in our women's health unit based on the American Heart Association's Blood Pressure Improvement Program. Simultaneously, a standard assessment of BP cuff sizing by arm measurement was implemented. We conducted a pre- and post-intervention assessment of nursing knowledge and confidence of BP measurement. Responses were analyzed using the $\chi^{2}$ test, two-sample $t$ test, ordinary least squares and logistic regression.

Results: A total of 145 nurses completed the pre- and 68 completed the post-intervention assessments. Participants answered $62 \%$ of pre- and $73 \%$ of post-intervention questions correctly $(p<.001)$. Before implementation, $86.9 \%$ of participants reported feeling very or extremely confident in obtaining an accurate BP measurement, increasing to $98.5 \%$ following $(p=.007)$. $73.8 \%$ of pre-intervention respondents reported feeling very or extremely confident in choosing an appropriate BP cuff compared to $96.3 \%$ post $(p<.001)$. Following implementation, confidence levels were similar irrespective of years in practice, years of experience at our hospital, and primary nursing unit.

Conclusions: A BP educational initiative and standardized BP cuff assessment increased nurses' knowledge and confidence in selecting the correct cuff size and obtaining accurate readings. Increased knowledge and confidence may lead to greater adherence to standardized BP assessment during peripartum admission, more accurate BP measurements, and improved management of hypertensive disorders in pregnancy.
\end{abstract}

Key Words: Maternal blood pressure, Hypertension, Obstetric nursing

\section{INTRODUCTION}

Hypertensive disorders remain a leading cause of maternal morbidity and mortality, complicating $10 \%$ of pregnancies worldwide. ${ }^{[1-5]}$ Hypertensive disorders of pregnancy in- clude chronic hypertension, gestational hypertension and preeclampsia with and without severe features. Obstetric hypertensive emergency, defined as sustained (> 15 minutes) severe range systolic blood pressure $>160 \mathrm{mmHg}$,

*Correspondence: Matthew K. Wagar; Email: mwagar@wisc.edu; Address: 202 S. Park St., Madison, WI 53715, USA. 
diastolic blood pressure $>110 \mathrm{mmHg}$ or both, further complicates hypertensive disorders of pregnancy and contributes to morbidity such as cerebral hemorrhage, stroke, and cardiovascular complications. ${ }^{[6,7]}$ The American College of Obstetricians and Gynecologists (ACOG) advocates for the prompt identification and treatment of obstetric hypertensive emergency within 60 minutes of diagnosis to reduce the risk of maternal morbidity and mortality. ${ }^{[6]}$ Implementation of the California Maternal Quality Care Collaborative (CMQCC) preeclampsia toolkit across 24 hospitals in the state of California demonstrated a reduction in severe maternal morbidity when obstetric hypertensive emergency was accurately identified, and treatment initiated within $60 \mathrm{~min}-$ utes of diagnosis. ${ }^{[8]}$

Accurate measurement of blood pressure in pregnancy is essential to guide diagnosis and management of hypertensive disorders and associated emergencies. ${ }^{[1,9]}$ The majority of deaths related to hypertensive disorders of pregnancy are considered preventable with timely and effective identification and intervention. ${ }^{[10]}$ Prompt diagnosis of hypertensive disorders of pregnancy relies on accurate blood pressure readings and adequate sizing of sphygmomanometer or automated oscillotonometric device arm cuffs. ${ }^{[11,12]}$ The importance of the accuracy of blood pressure cuff fit is underscored by the overestimation of blood pressure when a cuff is too small, and underestimation when a cuff is too large. ${ }^{[13-15]}$ ACOG, the American College of Cardiology (ACC) and the American Heart Association (AHA) advocate for standardized blood pressure assessments with an appropriately sized blood pressure cuff to establish an accurate diagnosis of hypertension. ${ }^{[6,11,16]}$ Despite these recommendations, lack of standardized blood pressure assessment protocols remains a contributor to preventable morbidity and mortality in the peripartum period. ${ }^{[17]}$

Hypertensive disorders of pregnancy are frequently assessed and diagnosed on initial presentation to obstetric triage units, most commonly by nursing staff. At UnityPoint Health (UPH)-Meriter, the obstetric tertiary care center of the University of Wisconsin - Madison, pregnant women are treated and admitted via an obstetric triage unit run by obstetriciangynecologists. We aimed to assess nursing knowledge and confidence regarding standardized blood pressure assessments before and after a quality improvement educational intervention designed to standardize blood pressure assessments based on the AHA blood pressure improvement program. ${ }^{[18]}$ We hypothesized that nursing knowledge regarding standard blood pressure assessment and confidence in obtaining accurate blood pressure measurements would increase following the intervention.

\section{MATERIALS AND METHODS}

This was a prospective quality improvement pilot project performed at UPH-Meriter from February 2020 to May 2020. This project was institutional review board exempt as reviewed by the UPH-Meriter Institutional Review Board and deemed quality improvement (QI). The Standards for Quality Improvement Reporting Excellence (SQUIRE) guidelines were followed in reporting this QI project. ${ }^{[19]}$ UPH-Meriter is the obstetric hospital of the University of Wisconsin Madison with more than 4,700 deliveries per year, and is a referral center for patients from Wisconsin, northern Illinois, and the upper peninsula of Michigan. The birthing center including obstetric triage, antepartum, labor \& delivery and postpartum units is run by obstetrician-gynecologists and staffed by obstetric and gynecology resident housestaff, family medicine physicians and resident housestaff, certified nurse midwives, and nurse practitioners.

The quality improvement initiative timeline involved several steps (see Figure 1). The nursing policy for obtaining blood pressure measurements in hypertensive disorders of pregnancy was reviewed and revised by the authors and nursing leadership. The educational initiative was developed based on the AHA blood pressure improvement program recommendations. ${ }^{[18]}$ An online presentation was created emphasizing the importance of standard blood pressure assessments, contributions of incorrect cuff sizing to misdiagnosis and morbidity, and best practices for obtaining an accurate blood pressure measurement. Standard positioning for blood pressure measurement was reviewed ${ }^{[6,18]}$ within the initiative including: 1) having the patient rest for 10 minutes or more before initial blood pressure measurement 2 ) empty bladder prior to obtaining measurement 3 ) having patient seated with legs uncrossed, feet and back supported with arm at heart level 4) avoid talking during measurement 5) confirmation of no caffeine or tobacco within $30 \mathrm{~min}$ utes of measurement 6) appropriate sized blood pressure cuff placed on bare arm confirmed by measurement of arm circumference (see Figure 2). Appropriate cuff sizes were determined based on recommendations put forth by the AHA and endorsed by ACOG (see Figure 3). ${ }^{[6,16]}$ Prior to the implementation of the intervention, no standard assessment for BP cuff selection existed at our institution. A simultaneous implementation of standard arm circumference measurement occurred with implementation of the educational initiative.

Nursing staff eligible for inclusion worked in any unit within the UPH-Meriter birthing center including obstetric triage, antepartum, labor \& delivery (L\&D) and postpartum. Preand post-intervention knowledge assessments and confidence surveys were administered one month prior to and one month following implementation, respectively. All departmental 
nursing staff were required to complete the educational initiative but were not required to complete knowledge assessments or confidence surveys. Pre- and post-intervention assessment included 9 knowledge questions and 5 confidence

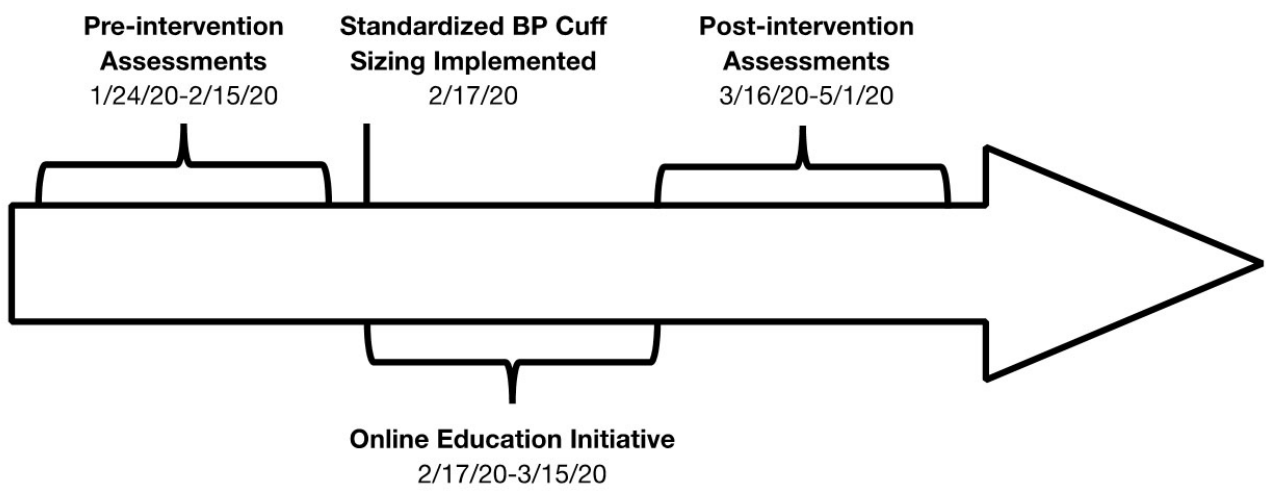
4.

Figure 1. Quality improvement intervention timeline 2020. BP: Blood Pressure

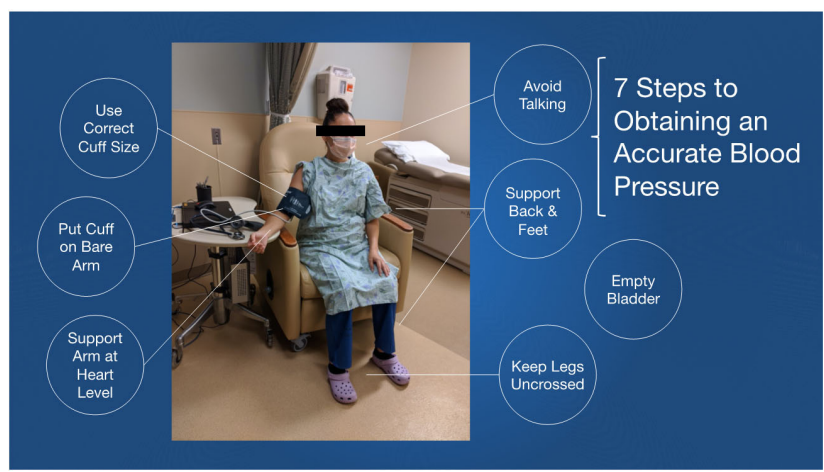

Figure 2. Steps for obtaining an accurate blood pressure assessment

- For an arm circumference of $22-26 \mathrm{~cm}$, the cuff should be small adult size: $12 \times 22 \mathrm{~cm}$.

- For an arm circumference of $27-34 \mathrm{~cm}$, the cuff should be adult size: $16 \times$ $30 \mathrm{~cm}$.

- For an arm circumference of $35-44 \mathrm{~cm}$, the cuff should be large adult size: $16 \times 36 \mathrm{~cm}$

- For an arm circumference of $45-52 \mathrm{~cm}$, the cuff should be adult thigh size: $16 \times 42 \mathrm{~cm}$

Figure 3. American Congress of Obstetricians and Gynecologists and the American Heart Association Recommendations for standard blood pressure cuff sizing

Staff characteristics including age, years in practice, years working at UPH-Meriter, years working in obstetrics, and primary birth center unit $(\mathrm{L} \& \mathrm{D}$, antepartum, triage v. postpartum) were obtained for pre- and post-intervention surveys. The a priori primary outcome was the percentage of correct questions scored on the pre- and post-intervention blood pressure measurement knowledge assessment. Preand post-assessments were not matched at the individual questions. Confidence items were developed following review of questions in consultation with the University of Wisconsin Survey Center (UWSC) and can be found in Figure

level. Secondary outcomes included sub-analysis of pre- to post-intervention confidence surveys by participant demographics. Our sample included all nursing staff employed in our hospital's birthing unit during the project implementation as all nursing staff were required to complete the required educational initiative. All statistical analyses were performed using Stata 16.1 (StataCorp LLC, College Station, TX). Data were summarized using means and standard deviations for continuous variables and proportions for categorical variables. Pre- and post-intervention variables were analyzed using the $\chi^{2}$ test for independence of association for categorical variables and the two-sample $t$ test for pre- and post-intervention knowledge scores. We used unadjusted ordinary least squares regression for the association between participant characteristics and knowledge scores. We used unadjusted logistic regression to test the association of participant characteristics with the confidence question; for purposes of these regressions, responses were dichotomized into very or extremely confident vs. not at all, a little or somewhat confident. A $p$ value of $<.05$ was considered statistically significant.

\section{RESULTS}

Two hundred and five staff nurses completed the educational initiative. One hundred and forty-five participants completed the pre-intervention knowledge assessment and confidence survey $(70.7 \%)$ and 68 completed the post-intervention assessment and survey (33.2\%). There were no significant differences between age of participants, years in practice, years working in obstetrics or years working at UPH-Meriter between the pre- and post-intervention groups. Staff completing the post-intervention survey were more likely to work 
on a postpartum unit compared to staff completing the pre- $(52(76.5 \%)$ v. $74(51 \%) p<.001)$. A summary of participant intervention knowledge assessment and confidence survey

characteristics is shown in Table 1.

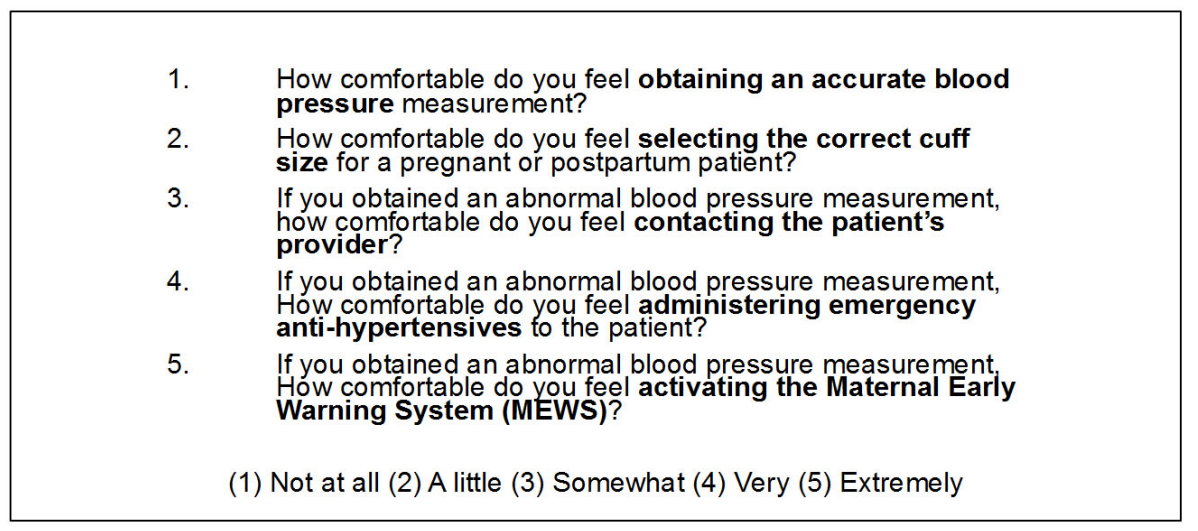

Figure 4. Pre- and post-intervention maternal blood pressure assessment confidence survey

Table 1. Respondent characteristics

\begin{tabular}{|c|c|c|c|}
\hline \multirow{3}{*}{ Characteristic } & Pre-intervention & Post-intervention & \multirow{3}{*}{$p$-value* } \\
\hline & $(\mathrm{N}=145)$ & $(\mathrm{N}=68)$ & \\
\hline & n (\%) & n (\%) & \\
\hline \multicolumn{3}{|l|}{ Age } & \multirow[t]{6}{*}{.740} \\
\hline $20-29$ & $51(35.7)$ & 25 (37.9) & \\
\hline $30-39$ & 47 (32.9) & $20(30.3)$ & \\
\hline $40-49$ & $20(14.0)$ & 13 (19.7) & \\
\hline $50-59$ & 17 (11.9) & $5(7.6)$ & \\
\hline $60+$ & $8(5.6)$ & $3(4.6)$ & \\
\hline \multicolumn{3}{|c|}{ Years in practice } & \multirow[t]{6}{*}{.611} \\
\hline$<$ one year & $10(7.0)$ & $6(8.8)$ & \\
\hline $1-2$ years & $10(7.0)$ & $7(10.3)$ & \\
\hline $2-5$ years & 38 (26.6) & $12(17.7)$ & \\
\hline $5-10$ years & $36(25.2)$ & $20(29.4)$ & \\
\hline$>10$ years & 49 (34.3) & $23(33.8)$ & \\
\hline \multicolumn{3}{|c|}{ Years at UPH-Meriter } & \multirow[t]{6}{*}{.816} \\
\hline$<$ one year & 25 (17.4) & $12(17.7)$ & \\
\hline $1-2$ years & $10(6.9)$ & $8(11.8)$ & \\
\hline $2-5$ years & $40(27.8)$ & $17(25.0)$ & \\
\hline $5-10$ years & 20 (13.9) & $10(14.7)$ & \\
\hline$>10$ years & $49(34.0)$ & 21 (30.9) & \\
\hline \multicolumn{2}{|l|}{ Years in OB } & & \multirow[t]{6}{*}{.987} \\
\hline$<$ one year & $20(14.0)$ & $9(13.4)$ & \\
\hline $1-2$ years & $14(9.8)$ & 8 (11.9) & \\
\hline $2-5$ years & $44(30.8)$ & $21(31.3)$ & \\
\hline 5-10 years & 25 (17.5) & $12(17.9)$ & \\
\hline$>10$ years & $40(28.0)$ & $17(25.4)$ & \\
\hline \multicolumn{3}{|l|}{ Primary unit } & \multirow[t]{3}{*}{.000} \\
\hline $\begin{array}{l}\text { Labor \& } \\
\text { delivery/Triage }\end{array}$ & $71(49.0)$ & $16(23.5)$ & \\
\hline Postpartum & $74(51.0)$ & $52(76.5)$ & \\
\hline
\end{tabular}

*Chi-square test for independence of association
The median pre-intervention knowledge score was 6 correct answers out of 9 questions, with a mean of $5.6 \pm$ 1.6, increasing to a median of 7 correct and mean of 6.6 \pm 1.6 post-intervention $(p<.001$ for difference in pre- vs. post-intervention means). The distribution of pre- and postintervention knowledge scores is shown in Figure 5. Performance on knowledge assessments were not significantly different across participant characteristics in unadjusted least squares regression for either pre- (see Table 2) or postintervention (see Table 3) assessments.

About $86.9 \%$ of participants reported feeling very or extremely comfortable with obtaining an accurate BP measurement prior to the intervention, increasing to $98.5 \%$ post initiative $(p<.001) .73 .8 \%$ of participants reported feeling very or extremely comfortable with selecting the correct BP cuff size for a pregnant or postpartum patient prior to the educational initiative, increasing to $95.6 \%$ following the intervention $(p<.001)$. There were no significant differences in comfort level pre- to post-intervention in regard to contacting physicians for abnormal BP finding, administering emergency anti-hypertensive agents or activating the maternal early warning system (MEWS).

In unadjusted logistic regressions, prior to the intervention, participants reported lower comfort levels in regard to contacting physicians for abnormal BP findings if they were younger (age 20-29), less experienced $(<2$ years in practice, $<2$ years of experience at our institution, or $<2$ years of experience in $\mathrm{OB}$ ), or worked primarily in our postpartum unit. Following the educational intervention, participants reported similar comfort levels in regard to contacting physicians for abnormal BP findings irrespective of age, experience, or primary nursing unit. 
Table 2. Regression of knowledge scores and comfort with BP on pre-intervention respondent characteristics

\begin{tabular}{|c|c|c|c|c|c|c|c|c|c|c|c|c|}
\hline \multirow{2}{*}{$\begin{array}{l}\begin{array}{l}\text { Pretest } \\
(\mathrm{N}=145)\end{array} \\
\begin{array}{l}\text { Characte- } \\
\text { ristic }\end{array}\end{array}$} & \multicolumn{2}{|c|}{$\begin{array}{l}\text { Number of knowledge } \\
\text { questions correct }\end{array}$} & \multicolumn{2}{|c|}{$\begin{array}{l}\text { Comfort obtaining } \\
\text { accurate BP }\end{array}$} & \multicolumn{2}{|c|}{$\begin{array}{l}\text { Comfort electing } \\
\text { right cuff size }\end{array}$} & \multicolumn{2}{|c|}{$\begin{array}{l}\text { Comfort contacting } \\
\text { provider }\end{array}$} & \multicolumn{2}{|c|}{$\begin{array}{l}\text { Comfort } \\
\text { administering } \\
\text { antihypertensive }\end{array}$} & \multicolumn{2}{|c|}{$\begin{array}{l}\text { Comfort activating } \\
\text { MEWS }\end{array}$} \\
\hline & $\begin{array}{l}\text { uCoeff } \\
\text { [95\% C.I.] }\end{array}$ & $p$ & $\begin{array}{l}\text { uOR } \\
\text { [95\% C.I.] }\end{array}$ & $p$ & $\begin{array}{l}\text { uOR } \\
\text { [95\% C.I.] }\end{array}$ & $p$ & $\begin{array}{l}\text { uOR [95\% } \\
\text { C.I.] }\end{array}$ & $p$ & $\begin{array}{l}\text { uOR }[95 \% \\
\text { C.I.] }\end{array}$ & $p$ & $\begin{array}{l}\text { uOR } \\
{[95 \% \text { C.I.] }}\end{array}$ & $p$ \\
\hline \multicolumn{13}{|l|}{ Age } \\
\hline $20-29$ & -reference- & & -reference- & & -reference- & & -reference- & & -reference- & & -reference- & \\
\hline 30-39 & $\begin{array}{l}0.25 \\
{[-0.34-0.84]}\end{array}$ & .400 & $\begin{array}{l}1.49 \\
{[0.51-4.38]}\end{array}$ & .470 & $\begin{array}{l}0.92 \\
{[0.29-2.97]}\end{array}$ & .889 & $\begin{array}{l}2.58 \\
{[1.12-5.94]}\end{array}$ & .026 & $\begin{array}{l}2.07 \\
{[0.77-5.54]}\end{array}$ & .148 & $\begin{array}{l}1.31 \\
{[0.53-3.23]}\end{array}$ & .560 \\
\hline $40+$ & $\begin{array}{l}0.22 \\
{[-0.38-0.81]} \\
\end{array}$ & .474 & $\begin{array}{l}2.03 \\
{[0.71-5.80]} \\
\end{array}$ & .184 & $\begin{array}{l}2.03 \\
{[0.71-5.80]} \\
\end{array}$ & .184 & $\begin{array}{l}3.73 \\
{[1.59-8.78]} \\
\end{array}$ & .003 & $\begin{array}{l}2.96 \\
{[1.12-7.81]} \\
\end{array}$ & .028 & $\begin{array}{l}1.70 \\
{[0.70-4.15]} \\
\end{array}$ & .243 \\
\hline \multicolumn{13}{|c|}{ Years in practice } \\
\hline$<2$ years & -reference- & & -reference- & & -reference- & & - reference- & & - reference- & & -reference- & \\
\hline 2-10 years & $\begin{array}{l}0.33 \\
{[-0.40-1.06]}\end{array}$ & .371 & $\begin{array}{l}2.10 \\
{[0.44-10.12]}\end{array}$ & .355 & $\begin{array}{l}1.74 \\
{[0.36-8.51]}\end{array}$ & .493 & $\begin{array}{l}2.68 \\
{[0.88-8.17]}\end{array}$ & .082 & $\begin{array}{l}2.89 \\
{[0.61-13.73]}\end{array}$ & .183 & $\begin{array}{l}2.00 \\
{[0.60-6.64]}\end{array}$ & .258 \\
\hline$>10$ years & $\begin{array}{l}0.53 \\
{[-0.24-1.29]}\end{array}$ & .178 & $\begin{array}{l}2.61 \\
{[0.52-13.00]}\end{array}$ & .243 & $\begin{array}{l}2.31 \\
{[0.46-11.63]}\end{array}$ & .311 & $\begin{array}{l}4.74 \\
{[1.48-15.17]}\end{array}$ & .009 & $\begin{array}{l}6.21 \\
{[1.29-29.78]}\end{array}$ & .022 & $\begin{array}{l}1.60 \\
{[0.45-5.63]}\end{array}$ & .464 \\
\hline \multicolumn{13}{|c|}{ Years at UPH-Meriter } \\
\hline$<2$ years & -reference- & & -reference- & & -reference- & & -reference- & & -reference- & & -reference- & \\
\hline 2-10 years & $\begin{array}{l}0.57 \\
{[-0.05-1.19]}\end{array}$ & .07 & $\begin{array}{l}1.20 \\
{[0.37-3.85]}\end{array}$ & .759 & $\begin{array}{l}0.79 \\
{[0.23-2.72]}\end{array}$ & .711 & $\begin{array}{l}3.88 \\
{[1.51-9.95]}\end{array}$ & .005 & $\begin{array}{l}4.27 \\
{[1.14-15.94]}\end{array}$ & .031 & $\begin{array}{l}4.08 \\
{[1.26-13.19]}\end{array}$ & .019 \\
\hline$>10$ years & $\begin{array}{l}0.51 \\
{[-0.14-1.15]}\end{array}$ & .122 & $\begin{array}{l}1.74 \\
{[0.54-5.54]}\end{array}$ & .351 & $\begin{array}{l}1.74 \\
{[0.54-5.54]}\end{array}$ & .351 & $\begin{array}{l}5.33 \\
{[2.01-14.14]}\end{array}$ & .001 & $\begin{array}{l}7.36 \\
{[1.98-27.36]}\end{array}$ & .003 & $\begin{array}{l}4.12 \\
{[1.25-13.61]}\end{array}$ & .020 \\
\hline \multicolumn{13}{|c|}{ Years in $\mathrm{OB}$} \\
\hline$<2$ years & - reference- & & -reference- & & -reference- & & - reference- & & -reference- & & - reference- & \\
\hline 2-10 years & $\begin{array}{l}0.43 \\
{[-0.19-1.05]}\end{array}$ & .169 & $\begin{array}{l}0.73 \\
{[0.26-2.09]}\end{array}$ & .560 & $\begin{array}{l}0.98 \\
{[0.31-3.14]}\end{array}$ & .977 & $\begin{array}{l}3.71 \\
{[1.42-9.71]}\end{array}$ & .007 & $\begin{array}{l}5.62 \\
{[1.22-25.90]}\end{array}$ & .027 & $\begin{array}{l}2.86 \\
{[0.98-8.40]}\end{array}$ & .055 \\
\hline$>10$ years & $\begin{array}{l}0.48 \\
{[-0.21-1.17]}\end{array}$ & .168 & $\begin{array}{l}1.12 \\
{[0.37-3.41]}\end{array}$ & .842 & $\begin{array}{l}1.68 \\
{[0.50-5.62]}\end{array}$ & .397 & $\begin{array}{l}7.71 \\
{[2.66-22.38]}\end{array}$ & .000 & $\begin{array}{l}13.12 \\
{[2.75-62.53]}\end{array}$ & .001 & $\begin{array}{l}2.70 \\
{[0.85-8.59]}\end{array}$ & .093 \\
\hline \multicolumn{13}{|c|}{ Primary unit } \\
\hline $\begin{array}{l}\text { Labor \& } \\
\text { delivery/ } \\
\text { Triage }\end{array}$ & -reference- & & -reference- & & -reference- & & -reference- & & -reference- & & -reference- & \\
\hline Postpartum & $\begin{array}{l}0.10 \\
{[-0.39-0.59]}\end{array}$ & .400 & $\begin{array}{l}0.87 \\
{[0.38-2.00]}\end{array}$ & .740 & $\begin{array}{l}0.52 \\
{[0.21-1.27]}\end{array}$ & .151 & $\begin{array}{l}0.49 \\
{[0.25-0.96]}\end{array}$ & .038 & $\begin{array}{l}0.29 \\
{[0.13-0.64]}\end{array}$ & .002 & $\begin{array}{l}1.47 \\
{[0.71-3.04]}\end{array}$ & .296 \\
\hline
\end{tabular}

Note. BP; blood pressure, OR: odds ratio, CI: confidence interval, MEWS; maternal early warning system, OB; obstetrics, UPH; UnityPoint Health.

Table 3. Post-intervention respondent characteristics and logistic regression

\begin{tabular}{|c|c|c|c|c|c|c|c|c|c|c|c|c|}
\hline \multirow{2}{*}{$\begin{array}{l}\begin{array}{l}\text { Posttest } \\
(\mathrm{N}=68)\end{array} \\
\begin{array}{l}\text { Characte- } \\
\text { ristic }\end{array} \\
\end{array}$} & \multicolumn{2}{|c|}{$\begin{array}{l}\text { Number of } \\
\text { knowledge questions } \\
\text { correct }\end{array}$} & \multicolumn{2}{|c|}{$\begin{array}{l}\text { Comfort obtaining } \\
\text { accurate BP }\end{array}$} & \multicolumn{2}{|c|}{$\begin{array}{l}\text { Comfort electing } \\
\text { right cuff size }\end{array}$} & \multicolumn{2}{|l|}{$\begin{array}{l}\text { Comfort } \\
\text { contacting } \\
\text { provider }\end{array}$} & \multicolumn{2}{|c|}{$\begin{array}{l}\text { Comfort } \\
\text { administering } \\
\text { antihypertensive }\end{array}$} & \multicolumn{2}{|c|}{$\begin{array}{l}\text { Comfort activating } \\
\text { MEWS }\end{array}$} \\
\hline & $\begin{array}{l}\text { uCoeff } \\
\text { [95\% C.I.] }\end{array}$ & $p$ & $\begin{array}{l}\text { uOR } \\
\text { [95\% C.I.] }\end{array}$ & $p$ & $\begin{array}{l}\text { uOR } \\
{[95 \% \text { C.I. }]}\end{array}$ & $p$ & $\begin{array}{l}\text { uOR } \\
\text { [95\% C.I.] }\end{array}$ & $p$ & $\begin{array}{l}\text { uOR } \\
{[95 \% \text { C.I. }]}\end{array}$ & $p$ & $\begin{array}{l}\text { uOR } \\
\text { [95\% C.I.] }\end{array}$ & $p$ \\
\hline \multicolumn{13}{|l|}{ Age } \\
\hline $20-29$ & -reference- & & -reference- & & -reference- & & -reference- & & -reference- & & -reference- & \\
\hline 30-39 & $\begin{array}{l}0.09 \\
{[-0.90-1.08]}\end{array}$ & .856 & $\begin{array}{l}1.71 \\
{[0.49-5.98]}\end{array}$ & .390 & $\begin{array}{l}1.08 \\
{[0.33-3.51]}\end{array}$ & .894 & $\begin{array}{l}0.92 \\
{[0.28-2.99]}\end{array}$ & .894 & $\begin{array}{l}0.28 \\
{[0.03-2.69]}\end{array}$ & .268 & $\begin{array}{l}1.45 \\
{[0.44-4.84]}\end{array}$ & .541 \\
\hline \multicolumn{13}{|c|}{ Years in practice } \\
\hline$<2$ years & -reference- & & - reference- & & -reference- & & -reference- & & -reference- & & -reference- & \\
\hline 2-10 years & $\begin{array}{l}0.15 \\
{[-0.92-1.22]}\end{array}$ & .781 & $\begin{array}{l}2.28 \\
{[0.52-9.92]}\end{array}$ & .272 & $\begin{array}{l}3.29 \\
{[0.83-12.98]}\end{array}$ & .089 & $\begin{array}{l}2.33 \\
{[0.62-8.77]}\end{array}$ & .208 & $\begin{array}{l}0.79 \\
{[0.13-4.92]}\end{array}$ & .797 & $\begin{array}{l}2.94 \\
{[0.68-12.73]}\end{array}$ & .149 \\
\hline$>10$ years & $\begin{array}{l}-0.02 \\
{[-1.14-1.11]}\end{array}$ & .976 & $\begin{array}{l}1.78 \\
{[0.38-8.37]} \\
\end{array}$ & .467 & $\begin{array}{l}1.45 \\
{[0.34-6.14]} \\
\end{array}$ & .617 & $\begin{array}{l}1.03 \\
{[0.25-4.16]}\end{array}$ & .968 & $\begin{array}{l}1.53 \\
{[0.25-9.27]} \\
\end{array}$ & .645 & $\begin{array}{l}1.18 \\
{[0.24-5.77]} \\
\end{array}$ & .841 \\
\hline \multicolumn{13}{|c|}{ Years at UPH-Meriter } \\
\hline $2-10$ years & $\begin{array}{l}-0.21 \\
{[-1.17-0.75]}\end{array}$ & .669 & $\begin{array}{l}2.06 \\
{[0.58-7.35]} \\
\end{array}$ & .264 & $\begin{array}{l}3.39 \\
{[1.00-11.57]}\end{array}$ & .051 & $\begin{array}{l}1.88 \\
{[0.58-6.06]}\end{array}$ & .294 & $\begin{array}{l}0.71 \\
{[0.13-3.94]} \\
\end{array}$ & .694 & $\begin{array}{l}2.40 \\
{[0.68-8.50]} \\
\end{array}$ & .175 \\
\hline$>10$ years & $\begin{array}{l}0.11 \\
{[-0.90-1.12]} \\
\end{array}$ & .826 & $\begin{array}{l}1.85 \\
{[0.48-7.06]}\end{array}$ & .370 & $\begin{array}{l}2.12 \\
{[0.59-7.66]} \\
\end{array}$ & .251 & $\begin{array}{l}1.36 \\
{[0.40-4.70]} \\
\end{array}$ & .624 & $\begin{array}{l}1.77 \\
{[0.36-8.65]} \\
\end{array}$ & .48 & $\begin{array}{l}1.5 \\
{[0.39-5.84]} \\
\end{array}$ & .559 \\
\hline \multicolumn{13}{|l|}{ Years in OB } \\
\hline$<2$ years & -reference- & & -reference- & & -reference- & & -reference- & & -reference- & & -reference- & \\
\hline 2-10 years & $\begin{array}{l}-0.25 \\
{[-1.22-0.72]}\end{array}$ & .609 & $\begin{array}{l}.11 \\
{[0.56-7.91]} \\
\end{array}$ & .267 & $\begin{array}{l}3.69 \\
{[1.05-12.96]}\end{array}$ & .051 & $\begin{array}{l}1.52 \\
{[0.47-4.95]}\end{array}$ & .489 & $\begin{array}{l}1.03 \\
{[0.17-6.31]} \\
\end{array}$ & .971 & $\begin{array}{l}2.71 \\
{[0.73-10.07]} \\
\end{array}$ & .137 \\
\hline$>10$ years & $\begin{array}{l}-0.59 \\
{[-1.17-1.05]}\end{array}$ & .916 & $\begin{array}{l}2.28 \\
{[0.52-9.99]} \\
\end{array}$ & .276 & $\begin{array}{l}1.68 \\
{[0.41-6.96]} \\
\end{array}$ & .474 & $\begin{array}{l}1.61 \\
{[0.41-6.24]} \\
\end{array}$ & .493 & $\begin{array}{l}3.13 \\
{[0.51-19.04]}\end{array}$ & .216 & $\begin{array}{l}1.35 \\
{[0.29-6.26]} \\
\end{array}$ & .698 \\
\hline \multicolumn{13}{|c|}{ Primary unit } \\
\hline $\begin{array}{l}\text { Labor \& } \\
\text { delivery/Tr } \\
\text { iage }\end{array}$ & -reference- & & -reference- & & -reference- & & -reference- & & -reference- & & -reference- & \\
\hline Postpartum & $\begin{array}{l}0.54 \\
{[0.37-1.46]}\end{array}$ & .239 & $\begin{array}{l}0.62 \\
{[0.20-1.96]}\end{array}$ & .420 & $\begin{array}{l}0.62 \\
{[0.20-1.91]}\end{array}$ & .402 & $\begin{array}{l}0.33 \\
{[0.10-1.10]}\end{array}$ & .071 & $\begin{array}{l}0.11 \\
{[0.03-0.44]}\end{array}$ & .002 & $\begin{array}{l}0.88 \\
{[0.28-2.82]}\end{array}$ & .833 \\
\hline
\end{tabular}




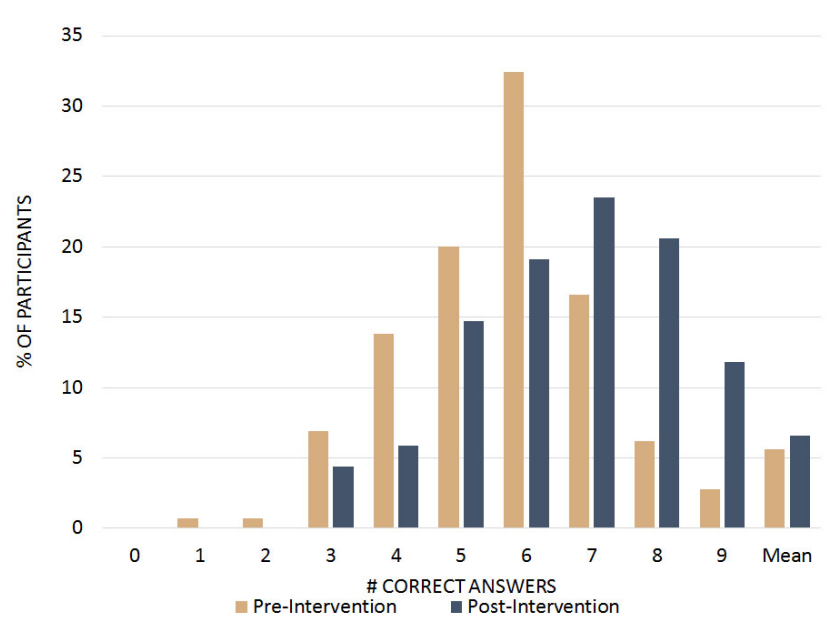

Figure 5. Number of correct blood pressure assessment answers by percentage of participants

Prior to the intervention, older (age 40 years and older) and more experienced nurses (in practice $>10$ years, $>2$ years of experience in obstetrics or $>2$ years of experience in our hospital) felt more comfortable administering emergency antihypertensive medications compared to those who were younger or less experienced. Following the intervention, staff reported similar levels of comfort in administering emergency antihypertensive medications regardless of age and experience. Notably, prior to our intervention, nurses who primarily staff our postpartum units reported lower comfort levels with administering emergency antihypertensive medications than nurses who primarily staff labor, triage, or antepartum, which was unchanged following the intervention $(\mathrm{p}=0.002)$.

Prior to our educational initiative, staff with $>2$ years of experience at our hospital reported higher comfort levels in activating our maternal early warning system (MEWS) compared to staff with $<2$ years of experience at our institution. Following the intervention, all participants reported similar comfort levels regardless of years at our institution. There were no significant differences in participant demographic factors and comfort level in obtaining an accurate BP or selecting the correct BP cuff size pre- or post-intervention. Complete regression results are found in Table 2 (pre-intervention) and Table 3 (post-intervention).

\section{Discussion}

The quality improvement initiative increased the primary outcome of nursing knowledge regarding obtaining an accurate maternal blood pressure assessment. In addition, staff comfort in obtaining accurate BP measurements and selecting the correct cuff size for pregnant patients increased significantly pre- to post-intervention for all participants.

While staff with less nursing experience reported lower comPublished by Sciedu Press fort levels when contacting physicians regarding an abnormal BP reading, administering emergency antihypertensive medications and activating the MEWS, these differences in comfort were no longer observed post-intervention. Participants who worked predominantly on our postpartum units reported lower comfort levels pre- and post-intervention administering emergency anti-hypertensive medications compared to staff who predominantly worked on our labor and delivery unit. We attribute this finding to the relative infrequency of severe range blood pressures encountered on our postpartum unit, as the majority of obstetric patients meeting criteria for a hypertensive disorder of pregnancy are identified antepartum or intrapartum and often have blood pressures stabilized by the time they reach the postpartum units. These findings suggest that an educational initiative aimed at increasing nursing knowledge and confidence regarding accurate BP measurement improves knowledge and confidence in addition to significantly improving comfort for staff with comparatively less experience.

Our quality improvement initiative adds to the evidence base for the 242 quality improvement opportunities (QIOs) identified by the California Pregnancy-Associated Mortality Review (CA-PAMR) in 2019. These QIOs were identified through case review of pregnancy related deaths in California from 2002-2007 specifically due to preeclampsia and eclampsia based on three of the four domains commonly used in maternal healthcare: Readiness, Recognition and Response. ${ }^{[17]}$ A few quality improvement initiatives focused on improving metrics within the Readiness and Response domains. ${ }^{[20-22]}$ Miller et al. demonstrated decreased time to administration of antihypertensive medications following implementation of an evidence-based quality improvement methodology focused on a nursing-initiated protocol for management of severe hypertension. ${ }^{[21]}$ Froelich et al. conducted a similar quality improvement initiative which failed to achieve its primary outcome of reducing time to achieve goal BP. ${ }^{[22]}$ Both studies suggest the elimination of error in obtaining accurate BP measurement may further reduce time to diagnosis and intervention. ${ }^{[21,22]}$

While our pilot project demonstrated an encouraging overall improvement in nursing knowledge and confidence, further evaluation for improvement in clinical metrics are needed. First, evaluation of time to antihypertensive administration should be evaluated to support the utility of increased knowledge and confidence of peripartum BP assessment. Next, the incidence of hypertensive diagnoses of pregnancy should be incorporated into quality improvement initiatives aimed at optimizing the accurate identification of abnormal blood pressures and hypertension in pregnancy. Further educational initiatives should focus on improving knowledge and 
familiarity with criteria for the diagnosis of hypertensive disorders of pregnancy. Finally, review of maternal morbidity and mortality should be incorporated into ongoing quality improvement initiatives to assess the effect on Recognition on maternal outcomes.

The strengths of this project include the multidisciplinary collaboration between physicians, nurses, resident physicians, and administration on a quality improvement initiative that led to increased knowledge and confidence. The prospective design adds strength to our results and the simplicity of the project's design makes it easy to replicate the initiative at another center and generalizable to obstetric populations similar to our own. Anticipated ethical issues arising from implementation of a standard blood pressure assessment include adherence to assessment across all care settings to ensure each patient receives equitable care. Additional considerations for equitable implementation include creating educational content accessible and obtainable for all staff irrespective of physical limitations such as sensory deficits. Limitations include the relatively lower response rate for our post-intervention survey and knowledge assessment, which

\section{REFERENCES}

[1] American College of Obstetricians and Gynecologists. Task Force on Hypertension in Pregnancy. Hypertension in pregnancy: report of the American College of Obstetricians and Gynecologists' Task Force on hypertension in pregnancy. Obstet Gynecol. 2013; 122(5): 1122-31.

[2] Say L, Chou D, Gemmill A, et al. Global causes of maternal death: a WHO systematic analysis. Lancet Glob Health. 2014; 2: e323-33. https://doi.org/10.1016/S2214-109X (14)70227-X

[3] Creanga AA, Syverson C, Seed K, et al. Pregnancy-related mortality in the United States, 2011-2013. Obstet Gynecol. 2017; 130: 366-73. PMid:28697109 https://doi.org/10.1097/A0G. 0000000000 002114

[4] Leeman L, Dresang LT, Fontaine P. Hypertensive disorders of pregnancy. Am Fam Physician. 2016; 93: 121-7.

[5] Hitti J, Sienas L, Walker S, et al. Contribution of hypertension to severe maternal morbidity. Am J Obstet Gynecol. 2018; 219: 405.e1-7. PMid:30012335 https://doi.org/10.1016/j.ajog. 2018.07 .002

[6] American College of Obstetricians and Gynecologists Chronic hypertension in pregnancy. ACOG Practice Bulletin No. 203. Obstet Gynecol. 2019; 133: e26-50. PMid:30575676 https://doi .org/ 10.1097/AOG.0000000000003020

[7] Too GT, Hill JB. Hypertensive crisis during pregnancy and postpartum period. Semin Perinatol. 2013; 37: 280-8. PMid:23916027 https://doi.org/10.1053/j. semperi.2013.04.007

[8] Druzin ML, Shields LE, Peterson NL, et al. Preeclampsia toolkit: improving health care response to preeclampsia. California Maternal Quality Care Collaborative toolkit to Transform Maternity Care. Developed under contract \#11- 10006 with the California Department of Public Health; Maternal Child and Adolescent Health Division; reduced our statistical power for that time-period, and the unpaired assessments. Given the relatively high confidence scores reported pre-intervention, assessment of maternal clinical outcomes is warranted to further understand secondary outcomes of our intervention. The application of our initiative to other obstetric care settings and centers different than our own may yield different results. Improvement in maternal outcomes following this intervention was also not assessed.

Safe and accurate assessment of maternal BP in an inpatient setting requires competency and confidence in the obtained measurements. Our project suggest that a simple educational initiative can improve knowledge and confidence in obtaining accurate blood pressure measurements and can improve the confidence of staff with less experience to levels comparable with their more seasoned peers. These findings highlight the need for ongoing quality improvement efforts aimed at optimizing assessment of maternal vital signs and recognizing abnormalities specific to pregnant women.

\section{CONFLICTS OF INTEREST DisClOSURE}

The authors declare that there is no conflict of interest. published by the California Maternal Quality Care Collaborative. 2013.

[9] Bello NA, Woolley JJ, Cleary KL, et al. Accuracy of blood pressure measurement devices in pregnancy: A systematic review of validation studies. Hypertension. 2018; 71(2): 326-335. PMid:29229741 https://doi.org/10.1161/HYPERTENSIONAHA.117.10295

[10] MacKay AP, Berg CJ, Atrash HK. Pregnancy-related mortality from preeclampsia and eclampsia. Obstet Gynecol. 2001; 97(4): 533-8. PMid:11275024 https://doi.org/10.1097/00006250-20010 4000-00011

[11] Pickering TG, Hall JE, Appel LJ, et al. Recommendations for blood pressure measurement in humans and experimental animals part 1: blood pressure measurement in humans: a statement for professionals from the Subcommittee of Professional and Public Education of the American Heart Association Council on High Blood Pressure Research. Hypertension. 2005; 45: 142-61. PMid:15611362 https://doi.org/10.1161/01.HYP.0000150859.47929.8e

[12] Eley VA, Christensen R, Kumar S, et al. A review of blood pressure measurement in obese pregnant women. International Journal of Obstetric Anesthesia. 2018; 35: 64-74. PMid:29954650 https://doi.org/10.1016/j.ijoa.2018.04.004

[13] Kho CL, Brown MA, Ong SLH, et al. Blood pressure measurement in pregnancy: the effect of arm circumference and sphygmomanometer cuff size. Obstet Med. 2009; 2: 116-20. PMid:27582825 https://doi.org/10.1258/om.2009.090017

[14] Schoenfeld A, Ziv I, Tzeel A, et al. Roll-over test - errors in interpretation, due to inaccurate blood pressure measurements. Eur J Obstet Gynecol Reprod Biol. 1985; 19: 23-30. https://doi.org/10.1 016/0028-2243(85) 90161-3 
[15] Geddes L, Tivey R. The importance of cuff width in measurement of blood pressure indirectly. Cardiovasc Res Cent Bull. 1976; 14: 69-79.

[16] Whelton PK, Carey RM, Aronow WS, et al. ACC/AHA/AAPA/ABC/ACPM/AGS/APhA/ASH/ASPC/NMA/PCNA guideline for the prevention, detection, evaluation, and management of high blood pressure in adults: a report of the American College of Cardiology/American Heart Association Task Force on Clinical Practice Guidelines [published erratum appears in J Am Coll Cardiol. 2018; 71: 2275-9. https://doi.org/10.1161/HYP.0000000000000076

[17] Morton CH, Seacrist MJ, VanOtterloo LR, et al. Quality Improvement Opportunities Identified Through Case Review of PregnancyRelated Deaths From Preeclampsia/Eclampsia. JOGNN - Journal of Obstetric, Gynecologic, and Neonatal Nursing. 2019; 48(3): 275-287. PMid:30980787 https : //doi .org/10.1016/j.jogn. 2019.02 .008

[18] American Heart Association. Target: BP Improvement Program. 2017.
[19] Davidoff F, Batalden P, Stevens D, et al. Publication guidelines for improvement studies in health care: Evolution of the SQUIRE project. Ann Intern Med. 2008; 149: 670-676. PMid:18981488 https: //doi.org/10.7326/0003-4819-149-9-200811040-00009

[20] von Dadelszen P, Sawchuck D, McMaster R, Douglas MJ, Lee SK, Saunders S, et al. The active implementation of pregnancy hypertension guidelines in British Columbia. Obstet Gynecol. 2010; 116: 659-66. PMid:20733449 https://doi .org/10.1097/AOG. 0b01 3e3181eb669d

[21] Miller MJ, Butler P, Gilchriest J, et al. Implementation of a standardized nurse initiated protocol to manage severe hypertension in pregnancy. J Matern Fetal Neonatal Med. 2020; 33(6): 10081014. PMid:30231657 https://doi .org/10.1080/14767058.2 018.1514381

[22] Froehlich RJ, Maggio L, Has P, et al. Improving obstetric hypertensive emergency treatment in a tertiary care women's emergency department. Obstet Gynecol. 2018; 132(4): 850-858. PMid:30130350 https://doi.org/10.1097/A0G.0000000000002809 